Miller, C. A. \& van Wagtendonk, W. J. (1956). J. gen. Microbiol. 15, 280-291

\title{
The Essential Metabolites of a Strain of Paramecium aurelia (Stock 47.8) and a Comparison of the Growth Rate of Different Strains of Paramecium aurelia in Axenic Culture
}

\author{
By C. A. MILlER* and W. J. van WAgtendonK \\ Department of Zoology, Indiana University, Bloomington, Inarana, U.S.A.
}

SUMMARY: Paramecium aurelia, var. 4, stock 47.8 (sensitive) grown in an axenic medium required the following amino acids: DL-tyrosine, L-phenylalanine, L-tryptophan, DL-methionine, DL-threonine, L-leucine, DL-isoleucine, L-lysine, L-histidine, L-arginine, and DL-serine. DL-valine, L-proline and glycine were synthesized at a slow rate. Tyrosine, methionine and serine were required by stock 51.7 (sensitive). Other essential metabolites required by stock $47.8(\mathrm{~s})$ were thiamine, riboflavin, folic acid, nicotinic acid, pantothenic acid, and possibly pyridoxal. 2,6diaminopurine inhibited growth at concentrations as low as $1-4 \mu \mathrm{g} . / \mathrm{ml}$. The growth rates (expressed as final population density/ml./transfer) of various stocks in identical media are different. An apparent correlation was found to exist between the growth rates and the chrcmosome number of these stocks, those with the largest chromosome number attaining the highest population density.

Paramecium aurelia was successfully established in axenic culture by van Wagtendonk \& Hackett (1949). The heat-sterilized medium was composed of equal parts of a $0.5 \%$ yeast autolysate (Basamin-Busch) and an autoclaved, $24 \mathrm{hr}$. culture of Aerobacter aerogenes in lettuce extract. Subsequent work led to the development of a medium consisting of salts, B-vitamins, a plant extract, a yeast extract, proteose-peptone and guanylic and cytidylic acids (van Wagtendonk, Miller \& Conner, 1952). The use of this medium made it possible to show that folic acid, riboflavin, thiamine and a steroid were absolute growth requirements for stock 57.1 (s) (van Wagtendonk, Conner, Miller \& Rao, 1953; Conner, van Wagtendonk \& Miller, 1953).

Additional stocks of Paramecium aurelia have now been established in axenic culture and maintained through more than a hundred serial subcultures. The specific amino acid and vitamin requirements of stock 47.8 (s), and a comparison of the growth of various stocks and their requirements are reported here.

\section{METHODS}

Five different stocks of Paramecium aurelia, variety 4, were examined in these experiments: $47.8(\mathrm{~s}), 51.7(\mathrm{~s})$ and its opposite mating type $51.8(\mathrm{~s})$, 32.7 (s), and 29.7 (s). None of these stocks contained kappa. Axenic cultures of stock $51.7(\mathrm{~s})$ were available from earlier experiments, while the other stocks

* Present address: Department of Biology, Wabash College, Crawfordsville, Indiana, U.S.A. 
were freed from associated bacterial forms by treatment with penicillin after the method of van Wagtendonk \& Hackett (1949). The organisms were cultured in $5 \mathrm{ml}$. of the test media in $15 \times 150 \mathrm{~mm}$. Pyrex tubes covered with metal caps. All experimental series were incubated at $27^{\circ}$ for 5 -day periods for each subculture. At the end of each 5 -day incubation period an inoculum $(0 \cdot 1-$ $0.3 \mathrm{ml}$.) was transferred to the next serial subculture. The total number of paramecia was determined by a direct count of measured samples from each tube and the counts from duplicate tubes were averaged to give the final population number. The growth obtained in the experimental tubes was expressed as a percentage of the control in each series, the control being arbitrarily taken as 100. All experiments were repeated at least once.

The method of preparation of the required unidentified factor(s) from yeast reported by van Wagtendonk et al. (1953) was modified. A 1/4 (w/v) suspension of Fleischmann's ' active dry' yeast was prepared by continuously stirring the yeast in distilled water at $50^{\circ}$ for $2-3 \mathrm{hr}$. The suspension was autoclaved for 20 min. at $120^{\circ}$ and centrifuged to collect a clear amber supernatant fluid. The supernatant fluid was again autoclaved in a section of cellulose dialysis tubing with one end left open to be tied off immediately after sterilization. This technique allowed for the exhaustive dialysis of large batches at room temperature without incurring contamination. The non-dialysable yeast fraction (NDF) was added aseptically to the basal medium given in Table 1. All the components of the medium may be autoclaved together in final concentration except the NDF and the B-vitamins which must be sterilized separately and added aseptically. All solutions were prepared with double distilled water; the $\mathrm{pH}$ value of the medium was $6 \cdot 8-7 \cdot 0$.

Table 1. Composition of basal medium for growth of Paramecium aurelia, var. 4 , stock 47.8

A non-dialysable yeast fraction (NDF) was added aseptically to this medium to give a complete medium. The B-vitamins were sterilized separately and added aseptically. The other components were autoclaved together in final concentration.

$\begin{array}{lclc} & \begin{array}{c}\text { Final } \\ \text { concn. } \\ (\mu \mathrm{g} \cdot / \mathrm{ml} .)\end{array} & & \begin{array}{c}\text { Final } \\ \text { concn. } \\ (\mu \mathrm{g} \cdot / \mathrm{ml} .\end{array} \\ \mathrm{MgSO}_{2} \cdot 7 \mathrm{H}_{2} \mathrm{O} & \mathbf{4 0 \cdot 0} & \text { Stigmasterol } & 0 \cdot 5 \\ \mathrm{Fe}\left(\mathrm{NH}_{4}\right)_{2}\left(\mathrm{SO}_{4}\right)_{2} \cdot 6 \mathrm{H}_{2} \mathrm{O} & 10 \cdot 0 & \text { Ca panthothenate } & 2 \cdot 0 \\ \mathrm{MnCl}_{2} \cdot 4 \mathrm{H}_{2} \mathrm{O} & 0 \cdot 1 & \text { Nicotinamide } & 2 \cdot 0 \\ \mathrm{ZnCl}_{2} & 0 \cdot 02 & \text { Pyridoxal. } \mathrm{HCl} & \mathbf{2 \cdot 0} \\ \mathrm{CaCl}_{2} \cdot 2 \mathrm{H}_{2} \mathrm{O} & 20 \cdot 0 & \text { Riboflavin } & 2 \cdot 0 \\ \mathrm{CuCl}_{2} \cdot 2 \mathrm{H}_{2} \mathrm{O} & 2 \cdot 0 & \text { Folic acid } & 1 \cdot 0 \\ \mathrm{FeCl}_{3} \cdot 6 \mathrm{H}_{2} \mathrm{O} & 0 \cdot 5 & \text { Thiamine } & 6 \cdot 0 \\ \mathrm{~K}_{2} \mathrm{HPO}_{4} & 570 \cdot 0 & \text { Nitrogen source: }(1 \text { \% proteose-peptone } & \\ \mathrm{KH}_{2} \mathrm{PO}_{4} & 570 \cdot 0 & \text { or amino acids as given in Table 2) } & \\ \text { Ethylenediamine tetraacetic } & 16 \cdot 0 & & \\ \text { acid } & & & \\ \text { Sodium acetate } & 570 \cdot 0 & & \\ \text { Sodium pyruvate } & 570 \cdot 0 & & \end{array}$

Conner \& van Wagtendonk (1955) showed that the steroid requirement of Paramecium aurelia stock 51.7, although fairly specific with regard to 
molecular configuration, may be satisfied by any one of several steroids. A preliminary report on the need for steroids by stock $\mathbf{4 7 . 8}$ was published by Conner, (1955), and a steroid requirement for $P$. multimicronucleatum has been shown by Johnson \& Miller (to be published). Thus, stigmasterol was included in all the media used in these experiments.

\section{RESULTS}

The requirement for a non-dialysable factor (NDF) from yeast

Studies are in progress to identify the unknown factor(s) present in the essential fraction from yeast. This component is an absolute requirement for all stocks of Paramecium aurelia thus far tested. Although the active yeast fraction used in these experiments remained quite complex, sufficient purification was obtained in the dialysis step alone to allow its use in the determination of several other essential metabolites. Upon dialysis of the initial aqueous extract from the yeast approximately $75 \%$ of the total solids were removed, and a tenfold purification of the activity on a weight basis was effected. The average weight of several NDF preparations was $12.5 \mathrm{mg} . / \mathrm{ml}$. The response of stosks 47.8 and 51.7 to different concentrations of the NFD, in the complete medium with amino acids is given in Fig. 1 . Concentrations below $0.5 \mathrm{mg} . / \mathrm{ml}$. would not support continued growth of the organisms. The non-dialysable yeast fraction gave a very faint ninhydrin reaction, a positive biuret reaction and a positive Benedict's test for reducing sugar after strong acid hydrolysis.

\section{Nitrogen requirements}

As shown in Table 3 the nitrogen requirements of Paramecium aurelia can be met by proteose-peptone. This material is satisfactory for routine growth and in its presence the requirements for stigmasterol, folic acid, riboflavin, thiamine and the NDF can be shown. However, it became desirable to replace proteose-peptone with known compounds in order to determine whether it was masking additional vitamin requirements, and to allow for a more specific analysis of the nitrogen requirements.

An acid hydrolysis of proteose-peptone was carried out by refluxing it for several hours with hydrochloric acid. The acid was removed by vacuum distillation and with the aid of Amberlite IR-45 (OH), whereupon the hydrolysate was resolved on a two-dimensional paper chromatogram. The following amino acids were identified: alanine, arginine, aspartic acid, glutamic acid, glycine, histidine, hydroxyproline, isoleucine, leucine, lysine, methionine, phenylalanine, proline, serine, threonine, tyrosine and valine. The presence of a small amount of cystine was also indicated.

Several preliminary experiments with stock 47.8 to test whether proteosepeptone could be replaced by its acid hydrolysate or by a mixture of the amino acids identified in the chromatogram indicated that either would permit growth provided tryptophan was also added. Different concentrations of the complete amino acid mixture and of some of the individual amino acids were tested to determine their approximate optimum concentrations. Table 2 lists the 


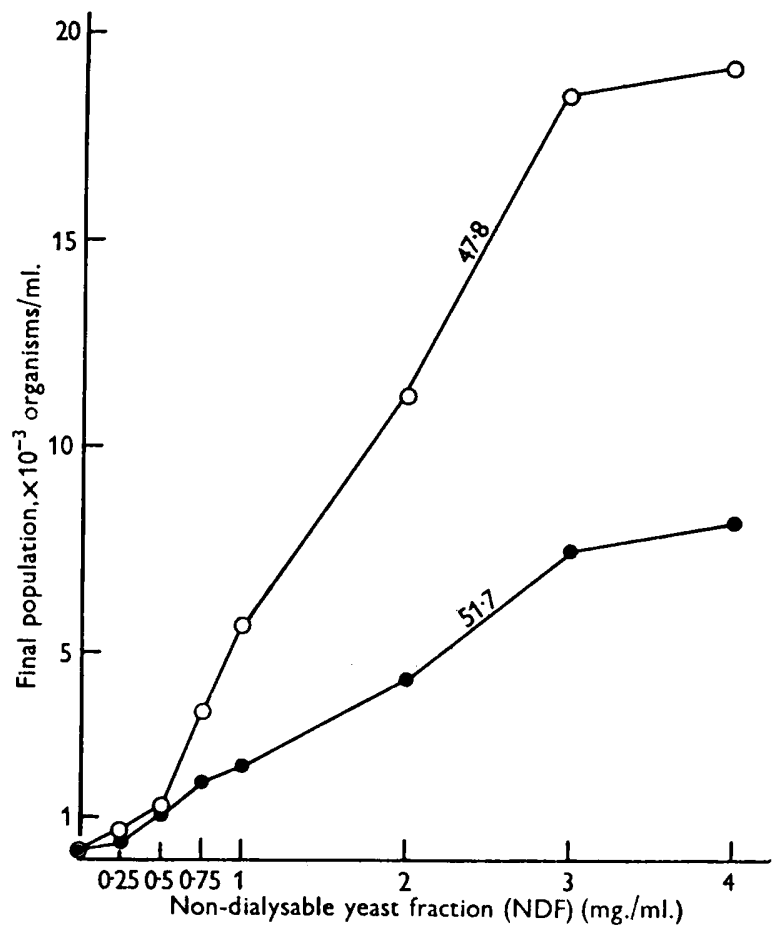

Fig. 1. The response of two stocks of Paramecium aurelia to varying concentrations of the non-dialysable yeast fraction (NDF) in the complete basal medium with amino acids. Concentrations of the NDF below $0.5 \mathrm{mg}$. $/ \mathrm{ml}$. would not support continued growth of the organisms. $O$, stock 47.8 ; 9 , stock 51.7 . The final population was determined 5 days after inoculation.

Table 2. Mixture of amino acids used to replace proteose-peptone in the basal medium for the growth of Paramecium aurelia, var. 4, stock 47.8

$\begin{array}{lclc} & \begin{array}{c}\text { Final concn. } \\ (\mu \mathrm{g} . / \mathrm{ml} .)\end{array} & & \begin{array}{c}\text { Final conen. } \\ (\mu \mathrm{g} . / \mathrm{ml} .)\end{array} \\ \text { L-Tryptophan } & \mathbf{5 0} & \text { DL-Serine } & \mathbf{2 0 0} \\ \text { DL-Methionine } & 150 & \text { L-Phenylalanine } & \mathbf{7 5} \\ \text { DL-Threonine } & 150 & \text { L(-)-Tyrosine } & \mathbf{5 0} \\ \text { DL-Isoleucine } & 150 & \text { L-Proline } & \mathbf{5 0} \\ \text { L-Leucine } & 150 & \text { L-Alanine } & \mathbf{2 5} \\ \text { L-Lysine } & 125 & \text { L-Aspartic acid } & \mathbf{5 0} \\ \text { L-Histidine } & \mathbf{5 0} & \text { L(+)-Glutamic acid } & \mathbf{7 5} \\ \text { L-Arginine } & 100 & \text { Glycine } & \mathbf{2 5} \\ \text { DL-Valine } & \mathbf{7 5} & \text { L(-)-Hydroxyproline } & 100 \\ & & \text { L-Cystine } & 50\end{array}$

concentrations which supported the most favourable growth. These concentrations were used throughout the experiments described below.

The results of experiments with organisms of stock 47.8 to compare the growth obtained with proteose-peptone, its hydrolysate, and amino acids as the nitrogen source are given in Table 3 . These results clearly demonstrate that: (1) free amino acids were utilized and served as the major nitrogen source; 
(2) the absence of specific single amino acids (tryptophan and tyrosine in this initial series) prevented or markedly limited continued growth; (3) the presence of one or more of the normally non-essential amino acids (alanine, aspartic acid, glutamic acid, glycine and proline) markedly stimulated growth. The absence of cystine or hydroxyproline did not impair growth.

Table 3. A comparison of the growth of Paramecium aurelia, var. 4, stock 47.8 in media containing different nitrogen sources

The nitrogen sources were tested in the complete basal medium using the NDF at a concentration of $2.5 \mathrm{mg} . / \mathrm{ml}$. A, $1 \%$ proteose peptone; B, an acid hydrolysate of proteose peptone (PP-H); C, PP-H plus tryptophan; D, a mixture of 18 amino acids contained in the PP-H plus tryptophan, as given in Table 2, (AA); E, AA minus hydroxyproline and cystine; F, AA minus tyrosine; G, AA minus alanine, proline, glycine, aspartic acid and glutamic acid.

Number of serial subcultures

$\begin{array}{lllllllll}1 & 2 & 3 & 4 & 5 & 6 & 7 & 8 & 9\end{array}$

Percentage organisms/unit volume of medium as compared with proteose peptone taken as $100 \%$

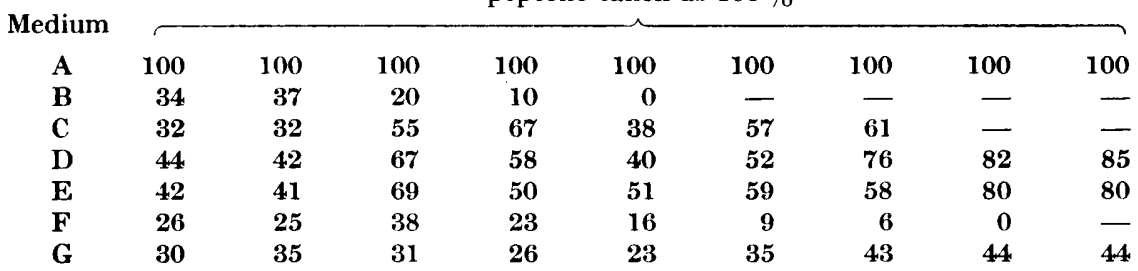

Using the single omission technique, extended series were designed to determine the need by organisms of stock 47 and 51 for each of the following amino acids: scrine, methionine, phenylalanine, tyrosine, tryptophan, threonine, isoleucine, leucine, lysine, histidine, valine, and arginine (Table 4). Both stocks require methionine, serine and tyrosine; stock 47.8 also showed the need for histidine.

Two to four mg. NDF/ml. were used in the medium for these initial experiments. The results indicated that either the NDF, at the concentration used, still contained a sufficient amount of protein to mask the detertion of all required amino acids, or that the organisms could synthesize most of the amino acids at a slow rate. Subsequent experiments with stock 47.8 , in which the concentration of NDF was decreased to $1 \mathrm{mg} . / \mathrm{ml}$. (w/v) confirmed the former hypothesis. The final population in the control (the complete amino acid medium) decreased thereby from an average of about 10,000 organisms/ $\mathrm{ml}$. to about $4000 / \mathrm{ml}$. (Fig. 1). However, the modification allowed for a more complete analysis of the amino acid requirements. Data obtained from these experiments are presented in Table 5. The single omission of each of the seventeen amino acids showed that eleven of them: tryptophan, methionine, threonine, isoleucine, leucine, lysine, histidine, arginine, serine, phenylalanine and tyrosine were absolute growth requirements for stock $\mathbf{4 7 . 8}$, for in each case no growth was obtained in the third serial subculture. 
The need for an exogenous source of valine, proline and glycine for optimum growth is also clear (Table 5). In the first transfer of Paramecium aurelia from a stock culture to a valine-less medium, growth was limited to $49 \%$ of the control followed by the stabilization of growth, through seven transfers at a level of $31 \%$ of the control. A more gradual growth decline was apparent in a medium lacking proline or glycine, and the number of organisms became stabilized at $50-40 \%$ of the control through seven subcultures. The omission of alanine, glutamic acid, or aspartic acid, singly, resulted in some growth decline in the initial transfers, but then all such cultures stabilized at numbers equal to or greater than the control. When proline, glycine, alanine, glutamic acid and aspartic acid together were omitted from the medium, growth was limited to about $40 \%$ of that obtained in the complete medium.

Table 4. A comparison of two stocks of Paramecium aurelia, var. 4, in a complete medium and in media lacking in amino acids

The complete medium was composed of the basal medium given in Table 1 plus the amino acid mixture given in Table 2 minus hydroxyproline and cystine. All media contained $4 \mathrm{mg} . / \mathrm{ml}$. of the non-dialysable yeast fraction (NDF).

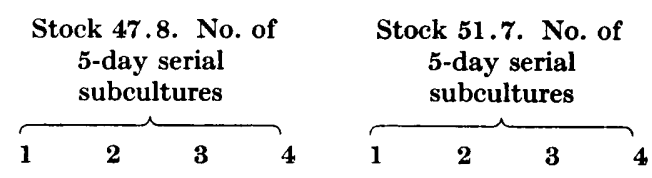

$\quad$ Medium
Complete
Complete
Minus phenylalanine
Minus tyrosine
Minus tryptophan
Minus methionine
Minus threonine
Minus isoleucine
Minus leucine
Minus lysine
Minus histidine
Minus arginine
Minus valine
Minus serine
Minus alanine, glycine,
proline, aspartic acid and
glutamic acid

Percentage organisms/unit volume of medium as compared with complete medium taken as $100 \%$

\begin{tabular}{|c|c|c|c|c|c|c|c|}
\hline 100 & 100 & 100 & 100 & 100 & 100 & 100 & 100 \\
\hline 50 & 43 & 41 & 78 & 89 & 86 & 45 & 81 \\
\hline 69 & 24 & 27 & 0 & 81 & 17 & 0 & - \\
\hline 65 & 30 & 45 & 63 & 89 & 62 & 65 & 100 \\
\hline 19 & o & $10^{*}$ & 0 & 45 & 0 & $17 *$ & 0 \\
\hline 42 & 38 & 32 & 50 & 78 & 69 & 45 & 57 \\
\hline 31 & 38 & 36 & 50 & 78 & 24 & 37 & 43 \\
\hline 54 & 43 & 52 & 53 & 100 & 45 & 50 & 65 \\
\hline 85 & 73 & 67 & 78 & 74 & 100 & 62 & 89 \\
\hline 58 & 70 & 47 & 0 & 96 & 100 & 89 & 89 \\
\hline 81 & 51 & 54 & 50 & 74 & 96 & 62 & 51 \\
\hline 62 & 73 & 54 & 75 & 81 & 79 & 70 & 81 \\
\hline 38 & 0 & $30^{*}$ & 16 & 59 & $\mathbf{0}$ & $47 *$ & 0 \\
\hline 44 & 48 & 49 & 45 & 87 & 79 & 78 & 74 \\
\hline
\end{tabular}

* Reinoculated.

\section{$B$-vitamin requirements}

The replacement of the complex proteose-peptone component of the earlier medium by amino acids made a re-examination of the B-vitamin requirements for Paramecium aurelia possible. Initial experiments with stock 47.8 in the amino acid medium, using high NDF concentrations, clearly showed that this stock required folic acid, riboflavin and thiamine. No indication for the requirement of choline, biotin or vitamin $B_{12}$ was apparent in any of the 
Table 5. Amino acid requirements of Paramecium aurelia, var. 4, stock 47.8

The complete medium was composed of the basal medium given in Table 1 plus the amino acid mixture given in Table 2 minus hydroxyproline and cystine. All media contained $1 \mathrm{mg} . / \mathrm{ml}$. of the non-dialysable fraction (NDF).

$\quad$ Medium
Complete
Minus tyrosine
Minus phenylalanine
Minus tyrosine minus
phenylalanine
Minus tyrosine $+\mathbf{3} \times$
phenylalanine
Minus tryptophan
Minus methionine
Minus threonine
Minus isoleucine
Minus leucine
Minus lysine
Minus histidine
Minus arginine
Minus serine
Minus valine
Minus proline
Minus glycine
Minus alanine
Minus aspartic acid
Minus glutamic acid
Minus proline, alanine, glycine,

No. of 5-day serial subcultures

$\overbrace{1}^{2} 43$

Percentage organisms/unit volume of medium as compared with complete medium taken as $100 \%$

\begin{tabular}{rrrr}
\hline 100 & 100 & 100 & 100 \\
35 & 0 & - & - \\
54 & 9 & 0 & - \\
48 & 3 & 0 & - \\
40 & 18 & 0 & - \\
& & & \\
32 & 9 & 0 & - \\
24 & 0 & - & - \\
30 & 6 & 0 & - \\
30 & 15 & 0 & - \\
35 & 9 & 0 & - \\
59 & 18 & 0 & - \\
32 & 3 & 0 & - \\
70 & 12 & 0 & - \\
12 & 0 & - & - \\
49 & 33 & 34 & $33^{*}$ \\
78 & 64 & 57 & $51^{*}$ \\
73 & 45 & 31 & $37^{*}$ \\
92 & 85 & 100 & $100^{*}$ \\
57 & 70 & 100 & $100^{*}$ \\
76 & 97 & 100 & $100^{*}$ \\
70 & 51 & 43 & $43^{*}$ \\
& & &
\end{tabular}

* Carried through three more transfers without change in the final population density.

experiments. That pantothenate, nicotinamide and pyridoxal might be required was, however, suggested by the inconsistent results which at times showed definite suboptimal growth in the absence of any one of these three vitamins. When using a lower NDF concentration $(0.75 \mathrm{mg}$. $/ \mathrm{ml}$.) a clearer response to the absence of these vitamins was given. Table 6 shows the results obtained upon the omission of each of these factors both singly and in combination. In addition to folic acid, riboflavin and thiamine, pantothenic acid and nicotinamide are essential metabolites for stock 47.8 , while a clearer indication for the requirement or perhaps the suboptimal synthesis of pyridoxal is apparent.

\section{Inhibition of growth by 2, 6-diaminopurine}

Hamilton (1953) reported that Paramecium aurelia, stock 51.7, grown on cultures of living Aerobacter aerogenes, directly utilized adenine, guanine and 2,6-diaminopurine for polynucleotide synthesis. Also a requirement for guanylic acid and cytidylic acid by Paramecium multimicronucleatum in axenic culture was reported by Johnson (1952). Young (1955) and Young \& 
van Wagtendonk (1956) found that $P$. aurelia, stock $51.7(\mathrm{~s})$, required a uridine diphosphate conjugate for growth in an axenic medium. In the course of testing several nucleic acid derivatives for growth-promoting activity it was observed that 2,6-diaminopurine inhibited the growth of organisms of stocks 51.7 and 47.8 in the range of $1-4 \mu \mathrm{g}$. $/ \mathrm{ml}$. The response of these stocks to increasing concentrations of 2,6-diaminopurine are given in Fig. 2.

Table 6. B-vitamin requirements of Paramecium aurelia, var. 4, stock 47.8

The complete medium was composed of the basal medium given in Table 1 plus the amino acid mixture given in Table 2 . All media contained $0.75 \mathrm{mg} . / \mathrm{ml}$. of the non-dialysable yeast fraction (NDF).

\begin{tabular}{|c|c|c|c|c|}
\hline & \multicolumn{4}{|c|}{ Number of 5-day serial subcultures } \\
\hline & $\mathbf{1}$ & $\mathbf{2}$ & $\mathbf{3}$ & 4 \\
\hline \multicolumn{5}{|c|}{$\begin{array}{l}\text { s compr } \\
\text { edium } t\end{array}$} \\
\hline Complete & 100 & 100 & 100 & 100 \\
\hline Minus folic acid & $\mathbf{0}$ & $0^{*}$ & $10^{*}$ & $\mathbf{0}$ \\
\hline Minus riboflavin & 34 & $\mathbf{0}$ & 29* & 0 \\
\hline Minus thiamine & 100 & 14 & $\mathbf{0}$ & $100 *+$ \\
\hline Minus pantothenic acid & $\mathbf{5 4}$ & 12 & $\mathbf{0}$ & $65 *$ \\
\hline Minus nicotinamide & 46 & 17 & $\mathbf{0}$ & $86 * t$ \\
\hline Minus pyridoxal & 88 & 35 & 49 & 348 \\
\hline Minus biotin & 79 & 93 & 100 & $100 \S$ \\
\hline Minus choline & 94 & 86 & 100 & 1008 \\
\hline
\end{tabular}

* Reinoculated. $†$ No growth in the next subculture. $\ddagger$ No growth in the third subculture. \$Carried through three additional subcultures without change in the final population density.

\section{Growth rate differences between stocks 47.8, 32.7, 51.7, 51.8, and 29.7 in axenic culture}

Differences in growth rate, and thus in final population densities, were immediately obvious among the different stocks when cultivated in an axenic medium. All of the stocks examined have been carried through more than 100 successive transfers and a direct comparison of the growth rates has been made from time to time. The differences in growth rate persist even in the most complex axenic medium employed. The rate of growth in an identical medium always followed the pattern : stock $47>$ stock $32>$ stock $51>$ stock 29 . Table 7 shows a direct comparison of these stocks carried simultaneously in the complete amino acid medium containing the optimum concentration of NDF. Both mating types of stock 51 were compared to determine whether a mating type difference might possibly effect a growth rate difference. An interesting comparison can be made between the difference in growth rate of these five stocks and the reported micronuclear chromosome numbers (Dippell, 1954) for these same stocks. The average rate of growth of the stocks parallels roughly the chromosome number. No difference in growth rate was apparent between the two mating types 7 and 8 . 


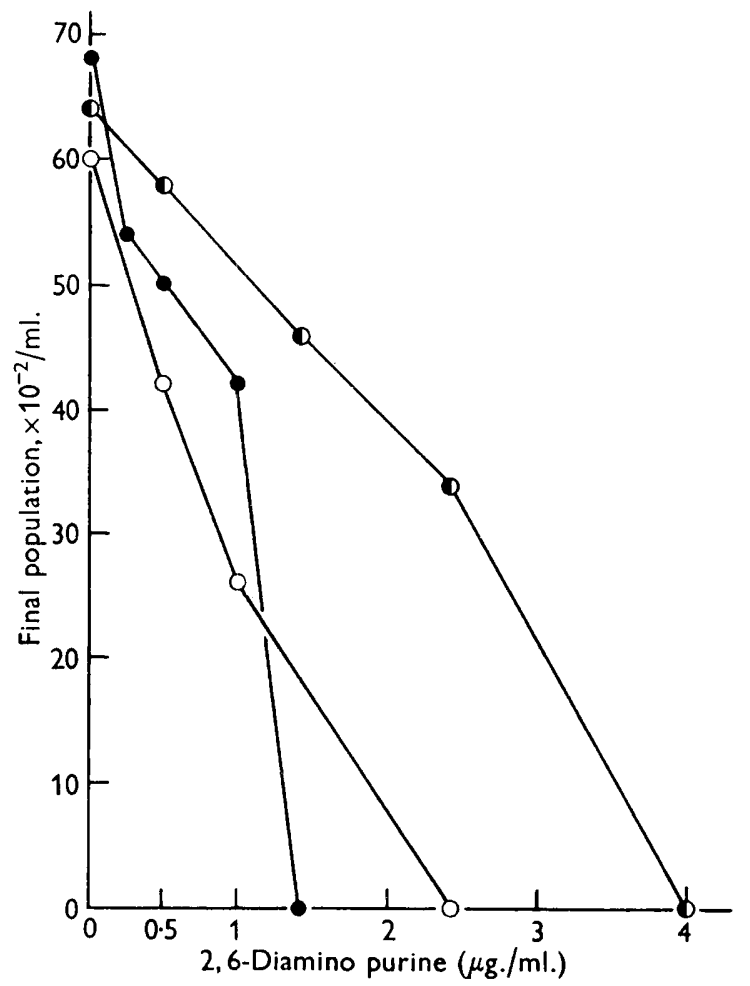

Fig. 2. Inhibition of the growth of Paramecium aurelia in various axenic media by 2,6 diaminopurine. Stock 47.8 in a proteose peptone-whole yeast extract $(4 \mathrm{mg} . / \mathrm{ml}$. $)$ medium; $O$, stock 47.8 in a proteose peptone + B-vitamins + NDF $(2 \mathrm{mg} . / \mathrm{ml}$.) medium; 0 , stock 51.7 in a proteose peptone $+\mathrm{B}$-vitamins + NDF $(2 \mathrm{mg} . / \mathrm{ml}$. $)$ medium. The final population was determined 5 days after inoculation.

Table 7. Differences in final population densities in successive subcultures and in chromosome numbers of five stocks of Paramecium aurelia, var. 4

The corresponding subcultures of the different stocks were grown simultaneously in the basal medium plus the amino acid mixtures shown in Table 2 and with a concentration of $1.5 \mathrm{mg} . / \mathrm{ml}$.

No. of 5-day serial subcultures

\begin{tabular}{|c|c|c|c|c|c|c|c|c|c|c|}
\hline \multirow{2}{*}{ Stock } & 1 & 2 & 3 & 4 & $\mathbf{5}$ & 6 & 7 & 8 & Mean & \multirow{2}{*}{$\begin{array}{c}\text { Chromosome } \\
\text { no. }\end{array}$} \\
\hline & \multicolumn{9}{|c|}{ Final population $\times 10^{-2}$ organisms $/ \mathrm{ml}$. } & \\
\hline 47.8 & 66 & 90 & 100 & 64 & 76 & 69 & 68 & 60 & 75 & $47-51$ \\
\hline 32.7 & - & - & - & 40 & 31 & 30 & 32 & 32 & 33 & $44-46$ \\
\hline 51.7 & 19 & 27 & 38 & 26 & 29 & 20 & 28 & 25 & 27 & $4,1-45$ \\
\hline 51.8 & - & 29 & 32 & 22 & 20 & 27 & 21 & 25 & 25 & $41-45$ \\
\hline 29.7 & 15 & 16 & 25 & 21 & 16 & 21 & 16 & 18 & 19 & $33-37$ \\
\hline
\end{tabular}

\section{DISCUSSION}

Specific amino acid requirements have been reported for only two other ciliates, Tetrahymena pyriformis (Kidder \& Dewey, 1951) and Glaucoma scintillans (Fuller, 1948). Although the basal requirements of Paramecium 
multimicronucleatum and $\boldsymbol{P}$. aurelia appear to be the same in crude medium, Johnson (1952) was unable to substitute free amino acids for Proteose-peptone in his sterile medium for $\boldsymbol{P}$. multimicronucleatum. The reason for this might be due to the use of an inadequate mixture of amino acids or to the low concentration of B-vitamins in the medium. Another factor which has contributed to the successful establishment of $P$. aurelia in an amino acid medium is the supplement of the essential sterol in a purified uniformly available form.

The replacement of Proteose-peptone with amino acids in the axenic medium for Paramecium aurelia constitutes a major step toward the goal of obtaining this organism in a complete chemically defined medium. The fact that growth falls to zero within the first few subcultures when any one of the reported eleven essential amino acids is absent from the medium indicates the lack of ability to synthesize these acids. The more than $75 \%$ diminution of growth in the absence of valine suggests that this amino acid can be synthesized only at a limiting rate by $P$. aurelia; this may also be the case for proline and glycine. A comparative nutritional study of several vertebrates has shown that valine is required by nearly all. Proline has been reported to be required by Trichomonas foetus (Weiss \& Ball, 1947), Glaucoma scintillans (Fuller, 1948), glycine by Trichomonas foetus, and by Aëdes aegypti (Goldberg \& de Meillon, 1948). The absolute requirement for serine by $P$. aurelia is paralleled in Tetrahymena pyriformis, strain E, as reported by Elliott (1949). A closer examination of the proper concentrations and metabolic interrelationships of the amino acid requirements of Paramecium aurelia must await the fuller understanding of other metabolites such as the carbon sources, salts, and other growth factors.

The inability of Paramecium aurelia to oxidize phenylalanine to tyrosine is concluded from the fact that an exogenous source of both amino acids is essential for continued growth. Most animals can meet their tyrosine needs when supplied with a source of phenylalanine, although apparently the reverse reaction does not occur; only tyrosine will spare phenylalanine in such cases. Until recently a one-step oxidation was postulated for the conversion of phenylalanine to tyrosine. However, studies of auxotrophic mutants of Escherichia coli, K 12, strain W (Simmonds, Dawling \& Stone, 1954) strongly support the view that the conversion of phenylalanine to tyrosine is indirect, the two amino acids being synthesized from a common precursor by reversible reactions.

The essential NDF component of the present medium remains complex. It contains polysaccharides, some protein, and apparently some non-dialysable vitamin conjugates. The yeast fraction contributes essential nucleic acid derivatives (Young, 1955; Young \& van Wagtendonk, 1956). This is supported by Johnson's (1952) demonstration of the need for guanylic and cytidylic acid in Paramecium multimicronucleatum.

Little is known of the requirements for recognized vitamins in ciliates other than Tetrahymena pyriformis. Kidder \& Dewey (1951) recorded that thiamine, riboflavin, the vitamin $B_{6}$ group, pantothenic acid, folic acid and nicotinamide are required for $T$. pyriformis. Colpoda duodenaria (Tatum, Garnjobst \& Taylor, 1942; Garnjobst, Tatum \& Taylor, 1943) requires relatively high

G. Microb. $x v$ 
concentrations of thiamine, riboflavin, pantothenic acid, nicotinamide and pyridoxine. Paramecium aurelia requires thiamine, riboflavin, pantothenic acid, nicotinamide and folic acid. The organism apparently can synthesize pyridoxal (and the other members of the $\mathbf{B}_{6}$ group) at a suboptimal rate.

The observations on the growth inhibition of two stocks of sterile Paramecium aurelia by 2,6-diaminopurine in a very low concentration are in direct contradiction with those reported by Hamilton (1953) for $P$. aurelia grown in living Aerobacter aerogenes cultures. Although further work must be done to clarify the phenomenon, this single observation emphasizes the need for employing axenic cultures for many of the studies designed to analyse protozoan metabolism.

The differences in growth rate of stocks $47.8,32.7,51.7,51.8$ and 29.7 cannot be satisfactorily explained. The difference is evident in each medium investigated and can therefore not be due to an unknown essential metabolite for the slower growing stocks. If the chromosome differences are indeed due to aneuploidy, as suggested by Dippell (1954), and if the apparent correlation between the chromosome number of the stocks and their rate of growth is valid, the following explanation might be offered. The stocks would differ by having different numbers of whole gene sets through the duplication of whole chromosomes or of parts thereof, rather than differing at the same single locus, or at a few loci through mutations. The differences in growth rates might then be explained by a greater or lesser synthetic capacity according to the number of like sets of genes present through ploidy in part of the genome.

This work was carried out under the auspices of a grant from the National Cancer Institute of the National Institutes of Health (no. C-2160(c)-), a grant from the National Science Foundation, a contract between the Office of Naval Research and Indiana University (no. NR 135-274) and grants from the Rockefeller Foundation and Indiana University. Partly based on a thesis presented by one of us (C. A. M.) in partial fulfilment of the requirements for the Ph.D. degree by the Indiana University.

\section{REFERENCES}

Conner, R. L. (1955). Growth requirements of Paramecium aurelia, variety 4, stock 47. Anat. Rec. 122, 427. (Abstract.)

Conner, R. L. \& Wagtendonk, W. J. van (1955). Steroid requirements of Paramecium aurelia. J. gen. Microbiol. 12, 31.

Conner, R. L., Wagtendonk, W. J. van \& Miller, C. A. (1953). The isolation from lemon juice of a growth factor of steroid nature required for the growth of a strain of Paramecium aurelia. J. gen. Microbiol. 9, 434.

Dippell, R. V. (1954). A preliminary report on the chromosomal constitution of certain variety 4 races of Paramecium aurelia. IX Congr. int. Genet. p. 1109.

Elliott, A. M. (1949). The amino acid requirements of Tetrahymena geleii (E.). Physiol. Zoöl. 22, 337.

Fuller, R. C. (1948). Studies on the biochemistry of Glaucoma scintillans (Master's thesis, Amherst College, Mass.).

Garnjobst, L., Tatum, E. L. \& Taylon, C. V. (1943). Further studies on the nutritional requirements of Colpoda duodenaria. J. cell. comp. Physiol. 21, 199.

Goldberg, L. \& De Meillon, B. (1948). The nutrition of the larva of Aëdes aegypti Linnaeus. 4. Protein and amino acid requirements. Biochem. J. 43, 379. 
Hamlton, L. (1953). Utilization of purines for nucleic acid synthesis in Chrysomonads and other organisms. Ann. N.Y. Acad. Sci. 56, 961.

Johnson, W. H. (1952). Further studies on the sterile culture of Paramecium. Physiol. Zoöl. 25, 10.

Kidder, G. W. \& Dewey, V. C. (1951). Biochemistry and Physiology of Protozoa, p. 323. New York: Academic Press.

Simmonds, S., Dawling, M. T., \& Stone, D. (1954). Metabolism of phenylalanine and tyrosine by Escherichia coli strain K-12. J. Biol. Chem. 208, 701.

Tatum, E. L., Garnjobst, L. \& Taylor, C. V. (1942). Vitamin requirements of Colpoda duodenaria. J. cell. comp. Physiol. 20, 211.

Wagtendonk, W. J. van, Conner, R. L., Miller, C. A. \& Rao, M. R. R. (1953). Growth requirements of Paramecium aurelia, var. 4, stock 51, sensitives and killers, in axenic medium. Ann. N.Y. Acad. Sci. 56, 929.

Wagtendonk, W. J. van \& Hacketr, P. L. (1949). The culture of Paramecium aurelia in the absence of other living organisms. Proc. nat. Acad. Sci. Wash. 35, 155.

Wagtendonk, W. J. van, Miller, C. A. \& Conner, R. L. (1952). Growth requirements of Paramecium aurelia, var. 4, stock $51.7(\mathrm{~s})$, in a medium free of other living organisms. Fed. Proc. 11, 302.

Weiss, E. D. \& BALL, G. H. (1947). Nutritional requirements of Tritrichomonas foetus with special reference to partially digested proteins. Proc. Soc. exp. Biol., N.Y. 65, 278.

Young, G. R. (1955). Growth requirement of Paramecium aurelia, var. 4, Stock 51.7 sensitives. Thesis, Indiana University.

Young, G. R. \& Wagtendonk, W. J. van (1956). A uridine phosphate conjugate as a growth requirement for Paramecium aurelia. Fed. Proc. 15, 390.

(Received 5 March 1956) 